$$
\frac{d^{2} \eta}{d \tau^{2}}+r^{-2} \eta+k T_{r}(\eta)=k \cos \tau
$$

which is the same as (7).

Similarly, by putting $\xi=(-1)^{r / 2} T_{r}(\eta)$ in (5) we can arrive at (10) and (11). Equation (12) and its solution can be established in the same manner by using a slightly different form of (5).

This approach shows that by a single transformation all the non-linear differential equations possessing simple subharmonics as their solutions can be derived from the linear problem which we understand much better. In other words, the simple subharmonic solutions might be considered merely as different representations of the same linear response solution in various frameworks. The physical significance, if any, of this transformation is not apparent at the present time.

Uniqueness of the solutions. There has been some discussion among interested people concerning the uniqueness of the simple subharmonics as steady-state solutions of (7). Since it is known that the linear response (6) is a unique steady state solution of (5), and since Eq. (7) and its simple subharmonic solutions can be derived from the linear case by a proper transformation, which is one-to-one when continuity conditions are imposed on the solutions, it may be concluded that these simple subharmonic solutions are unique so far as the steady state is concerned.

\title{
BIBLIOGRAPHY
}

1. R. M. Rosenberg, On the periodic solutions of the forced oscillator equation, Quart. Appl. Math. 15, 341 (1958)

2. R. M. Rosenberg, On the origin of subharmonic vibrations of odd orders, Proc., 2nd Midwest Conf. on Solid Mechanics, Purdue University, 1955

\section{Correction to my paper \\ ON THE DAMPED OSCILLATIONS EQUATION WITH VARIABLE COEFFICIENTS}

Quarterly of Applied Mathematics, XVI, 90-93 (1958)

Br E. V. LAITONE (University of California, Berkeley)

Equation (13) should have been written

$$
\phi(0)>\left\{\phi(0) u(0)^{2}+\left[u^{\prime}(0)+p(0) u(0) / 2\right]^{2}\right\}
$$

that is, the $u^{\prime}(0)$ should not be squared. Similarly the last equation on page 93 should contain only $\alpha^{\prime}(0)$ in the brackets. 